\section{Intraluminal fluorescence spectroscopy catheter with ultrasound guidance}

\author{
Douglas N. Stephens, ' Jesung Park, ${ }^{\text {a }}$ Yang Sun, ${ }^{a}$ \\ Thanassis Papaioannou, and Laura Marcu ${ }^{a, *}$ \\ a University of California, Davis, Department of Biomedical \\ Engineering, 451 Health Sciences Drive, Davis, \\ California 95616 \\ ${ }^{\mathrm{b}}$ Cedars-Sinai Medical Center, 8700 Beverly Boulevard, \\ Los Angeles, California 90048
}

\begin{abstract}
We demonstrate the feasibility of a timeresolved fluorescence spectroscopy (TRFS) technique for intraluminal investigation of arterial vessel composition under intravascular ultrasound (IVUS) guidance. A prototype 1.8-mm (5.4 Fr) catheter combining a side-viewing optical fiber (SVOF) and an IVUS catheter was constructed and tested with in vitro vessel phantoms. The prototype catheter can locate a fluorophore in the phantom vessel wall, steer the SVOF in place, perform blood flushing under flow conditions, and acquire high-quality TRFS data using 337-nm wavelength excitation. The catheter steering capability used for the coregistration of the IVUS image plane and the SVOF beam produce a guiding precision to an arterial phantom wall site location of $0.53 \pm 0.16 \mathrm{~mm}$. This new intravascular multimodal catheter enables the potential for in vivo arterial plaque composition identification using TRFS. () 2009 Society of PhotoOptical Instrumentation Engineers. [DOI: 10.1117/1.3146813]
\end{abstract}

Keywords: fluorescence spectroscopy; atherosclerosis; fluorescence lifetime; intravascular ultrasound.

Paper 08418LR received Nov. 30, 2008; revised manuscript received Apr. 14, 2009; accepted for publication Apr. 15, 2009; published online Jun. 3, 2009.

Catheter-based examination of arterial wall plaque characteristics is an important area of interest, especially as a means to study and diagnose atherosclerotic cardiovascular disease, which is considered the main cause of death in developed nations. ${ }^{1}$ We report the development of a new catheter-based approach for the detection of atherosclerotic plaques using intravascular ultrasound (IVUS) as an anatomical guidance method for the positioning of optical catheters capable of time-resolved fluorescence spectroscopy (TRFS).

TRFS itself, which can utilize both spectral and timedomain parameters, has been shown to detect elastin, collagen, lipids, and other sources of autofluorescence in normal and diseased arterial walls that exhibit inflammatory processes as well as to characterize the biochemical composition of human atherosclerotic plaques both ex vivo and in vivo. The comparison of various optically based methods for the analysis of plaques has produced a compelling rationale for a TRFS approach. On the basis of accuracy of plaque type identification, the following optical modalities have produced quality metrics, in terms of sensitivity and specificity against tissue histology, of: TRFS $(85 \%, 95 \%)^{2}$ and $(81 \%, 94 \%),{ }^{3}$ optical

*Address all correspondence to: Laura Marcu; E-mail: Imarcu@ucdavis.edu

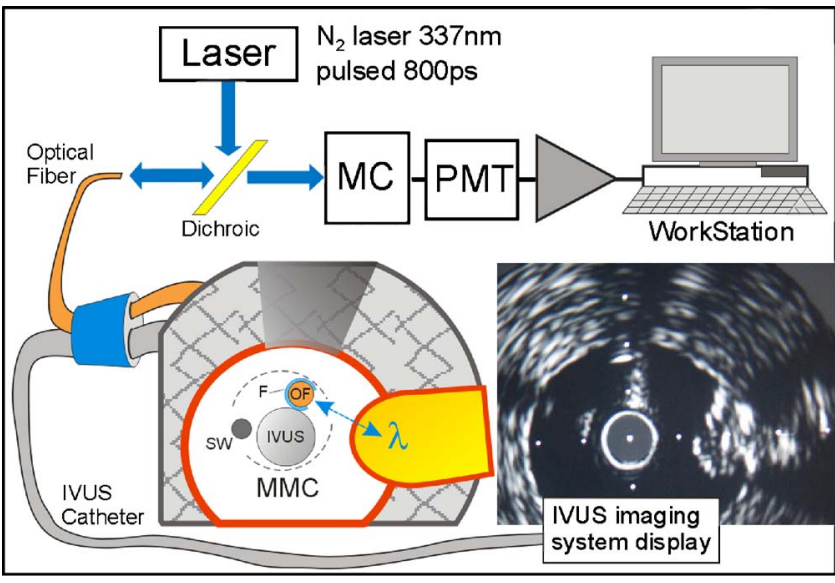

Fig. 1 A simplified system diagram showing the optical and ultrasound pathways to support the MMC. A cross section of the distal end of the MMC is shown in a phantom artery with a fluorophore target plug placed in the wall. IVUS imaging can clearly orient the SVOF (OF) within the water flushing lumen $(F)$ during the entire positioning procedure.

coherence tomography (OCT; ranges of 71 to $79 \%$ and 97 to $98 \%),{ }^{4}$ Raman spectroscopy (79 and $\left.85 \%\right),{ }^{5}$ and near-infrared (NIR) spectroscopy (ranges of 77 to $90 \%$ in sensitivity and $89 \%$ to $93 \%$ in specificity). ${ }^{6}$ OCT, $^{7}$ Raman spectroscopy, ${ }^{8}$ and NIR spectroscopy ${ }^{9}$ have been implemented in catheter-based devices for use in coronary arteries. The multimodal, multifunctional device described here can provide the opportunity for enhanced plaque diagnosis. The current approach will not only facilitate intravascular use of TRFS as the high-quality means to determine plaque composition but will also enable assessment of the plaque morphology or structure.

A practical means to implement the TRFS technique involves first an intravascular means of visual guidance for the optical fiber and second a steering method to coregister the optical fiber close to a particular arterial site in a moving blood environment. The novel approach described here addresses these issues to enable the optical and ultrasound modalities to operate in a beneficial and complementary way in a single multimodal catheter (MMC).

IVUS is now a commonly used catheter-based ultrasonic imaging technique to diagnose and guide interventional therapeutic procedures with real-time arterial cross-section images. It can provide specific lesion site identification and can provide direct guidance to an intraluminal location, and subsequent relocation, of specific lesion sites of interest. IVUS, as a catheter standard for ease of use ${ }^{10}$ is an obvious choice for the intraluminal guidance of the optical probe for use in obtaining TRFS data from sites of suspected vulnerable plaque.

The optical fiber itself (RoMack, Inc., Williamsburg, Virginia) is a customized side-viewing optical fiber (SVOF), which is shown in Fig. 1 as OF. It is a $400-\mu$ m-diam fused silica fiber (multimode, $\mathrm{NA}=0.22$ ) with a $45-\mathrm{deg}$ polished metalized mirror termination for beam deflection at 90 deg to the fiber axis. The optical fiber is coupled to a dichroic mirror, which is coupled to a prototype TRFS apparatus in our lab. This apparatus is similar to a TRFS system we previously

1083-3668/2009/14(3)/030505/3/\$25.00 @ 2009 SPIE 


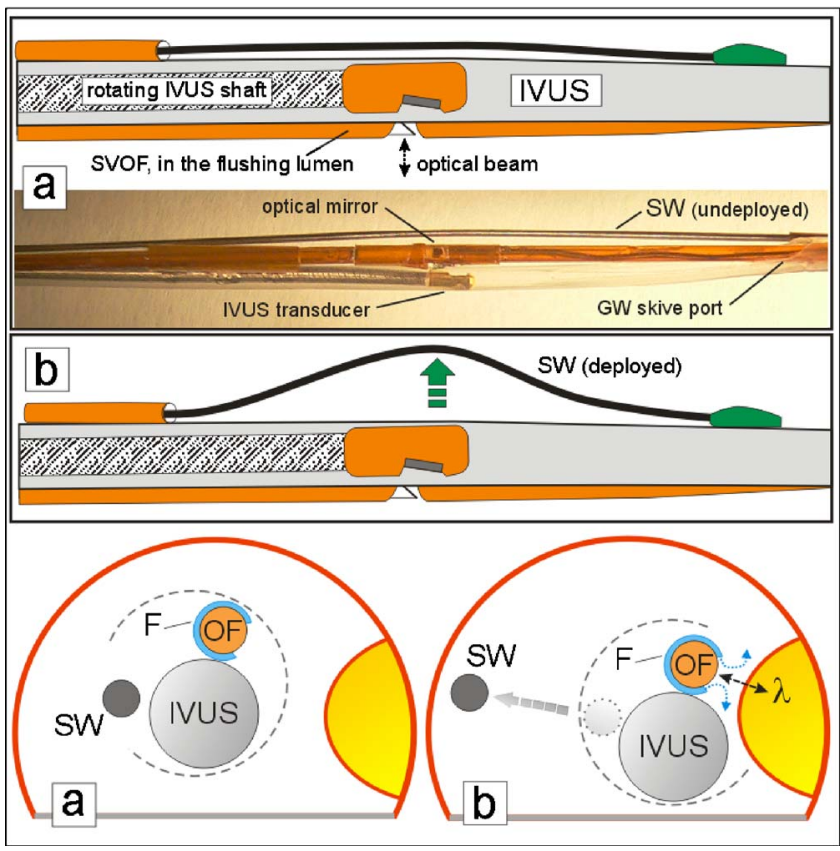

Fig. 2 Schematic and picture of the three-component construction of the MMC with deployment of lateral (steering) movement with the steering wire (SW). The normally compact MMC components are separated in the photo for clearer viewing. The SW in the undeployed state (a) is near the IVUS sheath and is mounted so that deployment (b) by pushing on the wire will move the MMC close to the fluorophore target in the phantom artery wall.

reported $^{11}$ and allows for wavelength-resolved fluorescence lifetime measurements. In brief, a pulsed $\mathrm{N}_{2}$ laser (MNL205-C, LTB Lasertechnik Berlin, Germany, $337.1 \mathrm{~nm}$, 800 -ps pulse width, $30-\mathrm{Hz}$ repetition rate) was used for autofluorescence excitation. The energy at the fiber tip was adjusted to $2 \mu \mathrm{J} /$ pulse by the adjustment of the iris in the fiber coupling port. The other major components of the optical system include a monochromator (MC; MicroHR, Horiba Jobin Yvon, Edison, New Jersey) for emission wavelength selection, a multichannel photomultiplier tube (MCP-PMT; R5916U-50, Hamamatsu, Bridgewater, New Jersey), a wideband amplifier (C-5594-12, 1.5-GHz bandwidth, Hamamatsu), and a fast digitizer (DPO 7254, 2.5-GHz bandwidth, 40 gigasamples/s, Tektronix, Richardson, Texas) with workstation. The singlefiber approach for TRFS data collection/analysis has been validated using fluorescence standard dyes and tissue endogenous fluorophores (elastin and collagen).

The IVUS imaging catheter used is a $3-\mathrm{Fr}(1 \mathrm{~mm})$ mechanical rotating type (SR Pro BSC, Fremont, California) catheter, which operates in the $30-$ to $40-\mathrm{MHz}$ range with its ClearViewUltra (BSC) imaging system.

The prototype MMC device has three major components, which are assembled in parallel combination to perform image-guided TRFS, as shown in cross section in Figs. 1 and 2. The 1-mm-diam IVUS catheter is the largest component. The side-viewing optical fiber (SVOF) is assembled inside a 0.7-mm-diam water flushing lumen; it occupies the position of the IVUS monorail guide wire and, in this prototype design, is adhesively fixed in position at the distal end of the IVUS catheter. The third component is the steering wire (SW) which is housed in its own outboard lumen adhesively at- tached to the IVUS shaft. An operator push of the SW serves to move the MMC laterally (Fig. 2) toward the luminal wall site of interest.

As shown in Figs. 1 and 2, the SVOF is rotated and adhesively positioned so that its optical mirror is directing light out of the fiber in a path at approximately $90 \mathrm{deg}$ to the IVUSSVOF axis. This allows the IVUS catheter to directly view the lumen wall target for TRFS investigation and also permits the mounting of the steering wire on the contralateral side of the beam, which allows more compact catheter assembly and permits more effective wire side-steering.

A 356- $\mu$ m-diam push-activated steering wire (SW; Fig. 2) is used to laterally direct the combination catheter to the wall; the proximal wire is confined in a small polyimide tube, which is adhesively fixed to the IVUS sheath. The small (0.7 $\mathrm{mm}$ diameter) fluid (saline) flushing tube surrounds the SVOF, except for a small saline exit window at the sideviewing optical beam path, to allow a local, low-volume water flush to achieve a large reduction in blood attenuation and scattering of the optical beam positioned close to the arterial wall.

Arterial phantoms made of polymethyl-methacrylate (PMMA) with wall-mounted fluorophore targets and ex vivo pig arteries were used to establish MMC performance metrics in a custom tank testing apparatus.

The optical beam width of the SVOF fundamentally determines the lower limit for optical spatial precision. The SVOF beam, exiting at $90 \mathrm{deg}$ to the MMC length axis, was measured to be $0.84 \mathrm{~mm}$ (FWHM) at a distance of $1 \mathrm{~mm}$ from the fiber surface. Based on experimental data, the spatial convolution of the SVOF beam in water and a standard $0.5-\mathrm{mm}$ fluorophore phantom were determined as a spatial reference standard to permit the subsequent coregistration accuracy of the MMC in an arterial phantom with the $0.5-\mathrm{mm}$ fluorophore mounted on the inside wall of the phantom. Fifteen measurements using only IVUS guidance produced a spatial positioning accuracy of the SVOF beam of $0.53 \pm 0.16 \mathrm{~mm}$.

An examination of the MMC performance in flowing whole blood conditions to assess (1) IVUS image guidance within either the phantom artery with a fluorescein isothiocyanate (FITC) fluorophore wall target or the pig femoral artery and (2) subsequent fluorescence spectroscopy was conducted in the following way. The arterial segment is mounted in the tank apparatus and blood introduced into the circulation system reservoir. The MMC catheter is then introduced through a hemostasis valve and advanced until the desired intraluminal wall position is in view with IVUS; the catheter is then rotated as needed to obtain the correct position (as shown in Figs. 1 and 2). The SW is then deployed to laterally shift the catheter for the optimal placement of the SVOF. With continuous low-volume $(0.07 \mathrm{ml} / \mathrm{s})$ saline flushing in the SVOF lumen, sample acquisitions of the intensity of fluorescence emission (470 nm for arterial data, $520 \mathrm{~nm}$ for FITC) are collected to gain feedback on good positioning. If the position yields a good fluorescence reading, the flushing is continued while the emission pulse transients across the entire fluorophore emission spectrum (360 to $560 \mathrm{~nm}, 5-\mathrm{nm}$ interval) are recorded by the fast digitizing oscilloscope. The discrete spectral intensities were reconstructed by integrating each pulse as a function of time. 


\section{JBO LETTERS}
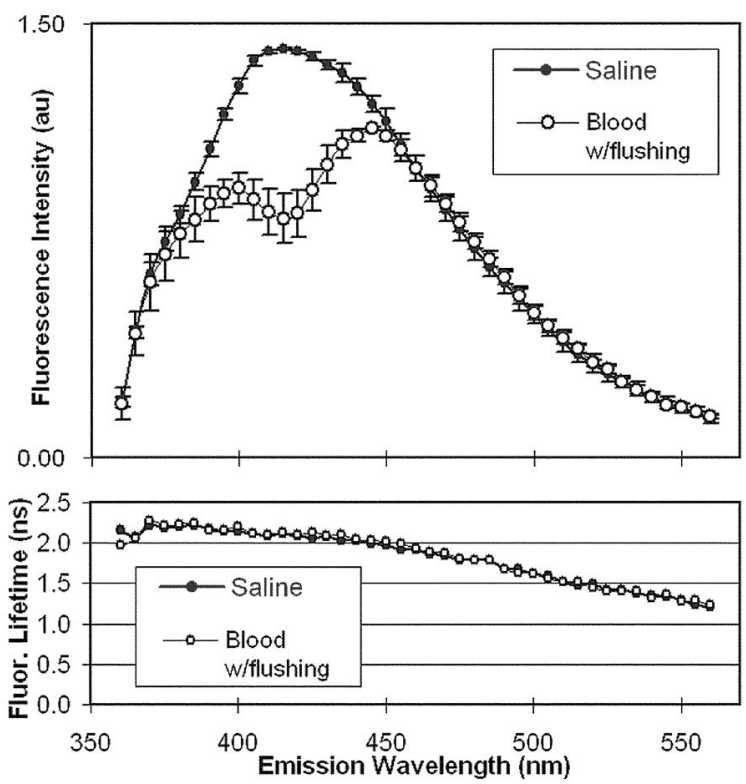

Fig. 3 Top panel: Comparison of MMC-based fluorescence spectra from an ex vivo pig femoral artery for moving whole blood $(N=7)$ conditions against the normalized spectral emission in diluted saline $(N=5)$. Bottom panel: The corresponding fluorescence lifetimes along the emission spectrum.

A pig femoral artery was used to test the effectiveness of saline flushing to diminish the optical attenuation effect of blood at $45 \%$ hematocrit. Using only IVUS for positional guidance, a calibration set of the TRFS data was collected first in water $(N=5)$, followed by TRFS data collections with multiple catheter withdrawal and repositioning steps in bovine blood $(N=7)$. The blood was circulated within the artery in a retrograde path with respect to the catheter direction at $5.9 \mathrm{~cm} / \mathrm{s}$. Saline flushing at $0.07 \mathrm{ml} / \mathrm{s}$ was continuously performed during the TRFS emission data collection across the emission spectrum at the rate of $0.77 \mathrm{~s} /$ wavelength (Fig. 3). We noted that the TRFS spectral intensity was modulated by the peak (at $415 \mathrm{~nm}$ ) in the blood absorbance, but the timeresolved data (average lifetime values) were not affected. As anticipated, this demonstrates that fluorescence lifetime measurements are more robust than intensity measurements in the presence of the endogenous absorbers such as blood. A fast TRFS system, which has been fully tested and recently reported, ${ }^{12}$ will be used in the future to obtain the data from arteries in less than 1 second.

This 5.4-F prototype MMC design demonstrates the feasibility of the IVUS guided approach for intravascular TRFS and provides the means for future studies in vivo in animal models of the effects of drug therapies on the atherosclerotic plaque composition. The next generation design will further integrate the optical fiber into the IVUS construction as a smaller, more flexible, and multifunction catheter with a size of $4.6 \mathrm{~F}(1.5 \mathrm{~mm})$.

\section{Acknowledgments}

The authors gratefully acknowledge Tat-Jin Teo at Boston Scientific Corp. for his cooperation and help with experimental materials. This work was supported in part by the NIH Grant No. R01 HL 067377 and the Philip Morris (USA and International) External Research Grant No. 07004732.

\section{References}

1. Y. Honda and P. J. Fitzgerald, "Frontiers in intravascular imaging technologies," Circulation 117(15), 2024-2037 (2008).

2. L. Marcu, Q. Y. Fang, J. A. Jo, T. Papaioannou, A. Dorafshar, T. Reil, J. Qiao, J. D. Baker, J. A. Freischlag, and M. C. Fishbein, "In vivo detection of macrophages in a rabbit atherosclerotic model by timeresolved laser-induced fluorescence spectroscopy," Atherosclerosis 181(2), 295-303 (2005).

3. L. Marcu, J. A. Jo, Q. Y. Fang, T. Papaioannou, T. Reil, J. Qiao, J. D. Baker, J. A. Freischlag, and M. C. Fishbein, "Detection of ruptureprone atherosclerotic plaques by time-resolved laser-induced fluorescence spectroscopy," Atherosclerosis 204(1), 156-164 (2009).

4. H. Yabushita, B. E. Bouma, S. L. Houser, H. T. Aretz, I. K. Jang, K. H. Schlendorf, C. R. Kauffman, M. Shishkov, D. H. Kang, E. F Halpern, and G. J. Tearney, "Characterization of human atherosclerosis by optical coherence tomography," Circulation 106(13), 16401645 (2002).

5. J. T. Motz, M. Fitzmaurice, A. Miller, S. J. Gandhi, A. S. Haka, L. H. Galindo, R. R. Dasari, J. R. Kramer, and M. S. Feld, "In vivo Raman spectral pathology of human atherosclerosis and vulnerable plaque," J. Biomed. Opt. 11(2), 021003-1 (2006).

6. P. R. Moreno, R. A. Lodder, K. R. Purushothaman, W. E. Charash, W. N. O'Connor, and J. E. Muller, "Detection of lipid pool, thin fibrous cap, and inflammatory cells in human aortic atherosclerotic plaques by near-infrared spectroscopy," Circulation 105(8), 923-927 (2002).

7. G. J. Tearney, S. A. Boppart, B. E. Bouma, M. E. Brezinski, N. J. Weissman, J. F. Southern, and J. G. Fujimoto, "Scanning single-mode fiber optic catheter-endoscope for optical coherence tomography," Opt. Lett. 21(7), 543-545 (1996).

8. Y. Komachi, H. Sato, K. Aizawa, and H. Tashiro, "Micro-optical fiber probe for use in an intravascular Raman endoscope," Appl. Opt. 44(22), 4722-4732 (2005).

9. S. Waxman, F. Ishibashi, and J. D. Caplan, "Rationale and use of near-infrared spectroscopy for detection of lipid-rich and vulnerable plaques," J. Nucl. Cardiol. 14(5), 719-728 (2007).

10. S. Waxman, F. Ishibashi, and J. E. Muller, "Detection and treatment of vulnerable plaques and vulnerable patients-novel approaches to prevention of coronary events," Circulation 114(22), 2390-2411 (2006).

11. Q. Y. Fang, T. Papaioannou, J. A. Jo, R. Vaitha, K. Shastry, and L. Marcu, "Time-domain laser-induced fluorescence spectroscopy apparatus for clinical diagnostics," Rev. Sci. Instrum. 75(1), 151-162 (2004).

12. Y. Sun, R. Liu, D. S. Elson, C. W. Hollars, J. A. Jo, J. Park, Y. Sun, and L. Marcu, "Simultaneous time- and wavelength-resolved fluorescence spectroscopy for near real-time tissue diagnosis," Opt. Lett. 33(6), 630-632 (2008). 